\title{
Retroperitoneal Spindle Cell Tumor: A Case Report
}

\author{
Hao Hua, Zhiwei He, Linhan Lei, Huahua Xie, Zilei Deng, Zili Cheng, Shi Zuo, Chengyi Sun \\ and Chao $\mathrm{Yu}^{*}$
}

Department of Hepatic-Biliary-Pancreatic Surgery, The Affiliate Hospital of Guizhou Medical University, Guiyang, China

Spindle cell tumor is very rare. Herein, we report a case of retroperitoneal spindle cell tumor in a 52-year-old female. The patient first presented with a complaint of persistent pain in the right upper abdomen. In the follow-up, a CT scan was performed and showed a retroperitoneal soft tissue density mass measuring $11 \mathrm{~cm}$ in diameter. Then, a subsequent operation was performed, and we completely removed the tumor and partially invaded lesions. The tumor was histologically diagnosed as a spindle cell tumor. Therefore, it is imperative for us to enhance the understanding of this seldom found tumor. Surgery remains the best option for treatment.

Keywords: retroperitoneal, spindle cell tumor, myofibroblastoma, surgery, case report

\section{OPEN ACCESS}

Edited by:

Hakan Kulacoglu,

Ankara Hernia Center, Turkey

Reviewed by:

Xianbing Kong,

First Affiliated Hospital of Chongqing

Medical University, China

Fei Xie,

The First People's Hospital of

Neijiang, China

*Correspondence:

Chao Yu

yuchao2002@gmc.edu.cn

Specialty section:

This article was submitted to

Visceral Surgery,

a section of the journal

Frontiers in Surgery

Received: 26 August 2021 Accepted: 08 November 2021 Published: 15 December 2021

Citation:

Hua H, He Z, Lei L, Xie H, Deng Z, Cheng Z, Zuo S, Sun C and Yu C (2021) Retroperitoneal Spindle Cell

Tumor: A Case Report.

Front. Surg. 8:764901.

doi: 10.3389/fsurg.2021.764901

\section{INTRODUCTION}

Various types of human tumors can present as spindle cells, such as poorly differentiated epithelial-derived tumors, gastrointestinal stromal tumors, mesenchymal tumors, and neurogenic tumors $(1,2)$. Spindle cell tumor, characterized histologically by a mixture of fat cells and fibroblastlike spindle cells in a matrix of collagen and mucoid material, is rare, and its incidence is low and can occur in human soft tissue, bone, or in any part of the human body, such as the retroperitoneal space. Its morphological appearance can be carcinomatous or neoplastic. Because of its rarity, there have been no adequate studies on its clinicopathological characteristics and diagnosis scheme. Herein, we are present a case of retroperitoneal spindle cell tumor to raise awareness on this seldom found tumor.

\section{CASE PRESENTATION}

A 52-year-old female patient presented with a complaint of persistent pain of 1 month duration in the right upper abdomen. There was no history of previous abdominal trauma, bleeding, or family history of cancer. On clinical examination, there was slight tenderness in the right upper abdomen. Serum $\alpha$-fetoprotein, carcinoembryonic antigen, carbohydrate antigen 125, and carbohydrate antigen 19-9 were normal. There was no obvious clinical history of malignancy. Enhanced upper abdominal computerized tomography (CT) showed a soft tissue density mass $11 \mathrm{~cm}$ in diameter (Figure 1). Therefore, we speculated that the retroperitoneal tumor was a benign tumor. Additionally, pathological results of pre-operative puncture were inflammatory myofibroblastoma and nodular fasciitis. Then, we chose to perform surgery.

The findings of intraoperative exploration revealed that the tumor was involved in the right hepatic artery, main portal vein, right portal vein, gallbladder, gastric antrum, duodenum, and head of pancreas. According to the results, we performed pancreaticoduodenectomy, perihilar hepatectomy, neoplasty of high bile duct, partial resection and repair of the main portal vein, partial resection and repair of the right portal vein, repair of the right hepatic artery, and 

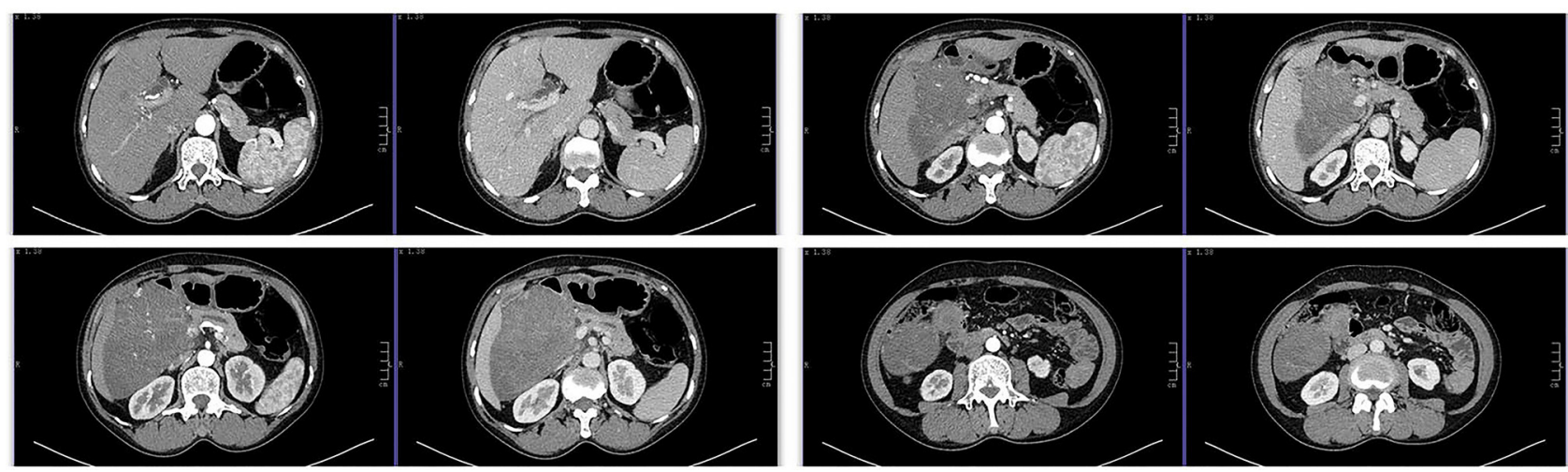

FIGURE 1 | Computed tomography (CT) scan showed a retroperitoneal soft tissue density mass, $11 \mathrm{~cm}$ in size, which is closely related to the liver, first and third hepatic portals, portal vein, hepatic artery, and pancreas.

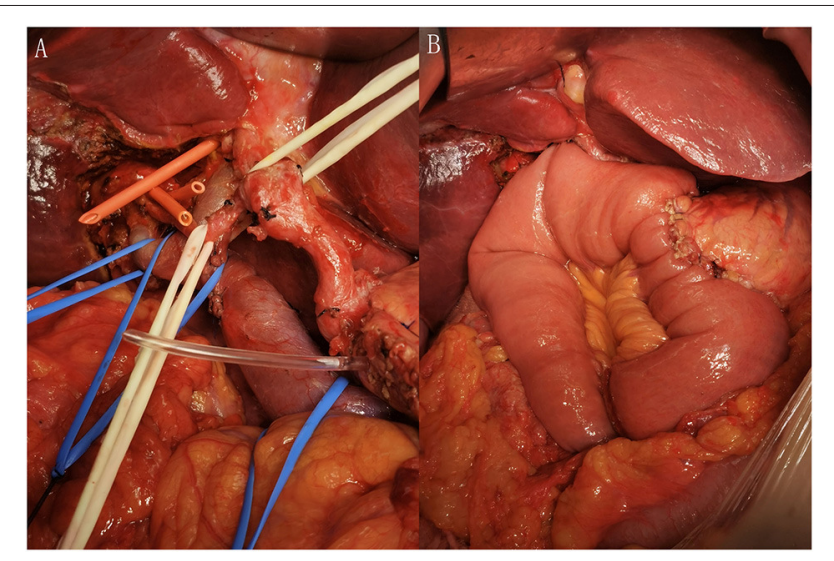

FIGURE 2 | (A) After removal of the tumor from the abdominal cavity and (B) after the reconstruction of digestive tract.

reconstruction of the digestive tract (Figure 2). Finally, the operation was successful, lasting $7 \mathrm{~h}$, and the intraoperative bleeding was $350 \mathrm{ml}$. The patient recovered well after the surgery.

The tumor was solitary and solid with an off-white crosssection (Figure 3). The actual size of the tumor was $\sim 17 \times 12 \mathrm{~cm}$. Microscopically, the tumor was predominantly made of spindle cells, and the margin of resection was negative (Figure 4). The tumor was diagnosed as a spindle cell tumor depended on these consequences.

\section{DISCUSSION}

To the best of our knowledge, soft tissue tumors usually occur in the limbs. Therefore, abdominal soft tissue tumors, especially retroperitoneal spindle cell tumors, are uncommon (3). Recently, there are still insufficient clinical research studies on spindle cell tumors. Spindle cell tumors, first reported by Weiss and Enzinger in 1896, originate from a variety of types of tumors, such as fibrosarcoma, gastrointestinal stromal tumor, and intraabdominal desmoid tumor (4). Most of them occur at the young age of 20-40 years, occasionally appear in child, and their incidence has no significant differences between males and females $(5,6)$. In 1994, the WHO officially classified spindle cell tumors as soft tissue neoplasms.

Due to the diversity of pathological morphology, it was once named after inflammatory pseudotumor. A previous article has reported that the biological behavior of the tumor cells is inert, and is associated with distant metastasis, and that their prognosis is well (2). Clinical examination, imaging, and histology are non-specific in the evaluation of the uncommon tumor and distinguish it from other solid masses. Thus, pathological and immunohistochemical tests are critical to the definitive diagnosis of this kind of tumor. $\beta$-Catenin has an imperative role in the development of the differential diagnosis of spindle cell neoplasms, particularly in the abdomen (710). In a prior study, Torres reported that $\beta$-catenin nuclear staining is probably the single most vital feature and is fundamental for the diagnosis (11). Meanwhile, the study by Carlson indicated that nuclear immunoreactivity for $\beta$-catenin is supportive for the diagnosis of this kind of lesion (12). Also, immunohistochemical staining of CD34 and CD117 antibodies can be performed for differential diagnosis (13). Another research found that the application of Ki-67 for the identification of tumor cells with spindle cell morphologic characteristics of smooth muscle cells and fibroblasts is of great importance (14). In our case, the diagnosis still requires pathological and immunohistochemical confirmation. This is a unique case report not only because retroperitoneal spindle cell tumors are extremely rare, but also because the tumor was diagnosed as fibromatosis. However, identification of additional cases and further research studies on the diagnosis of spindle cell tumor are necessary.

The management of retroperitoneal spindle cell tumors is complicated and based on their clinical biological behavior. In this case, CT is effective in detecting the soft tissue density mass located in enterocoelia, which was suspected of local lesion. The pathological results of pre-operative puncture are different from the post-operative results mainly not only because of its rarity but also plenty of neoplasms have overlapping cytomorphologic 


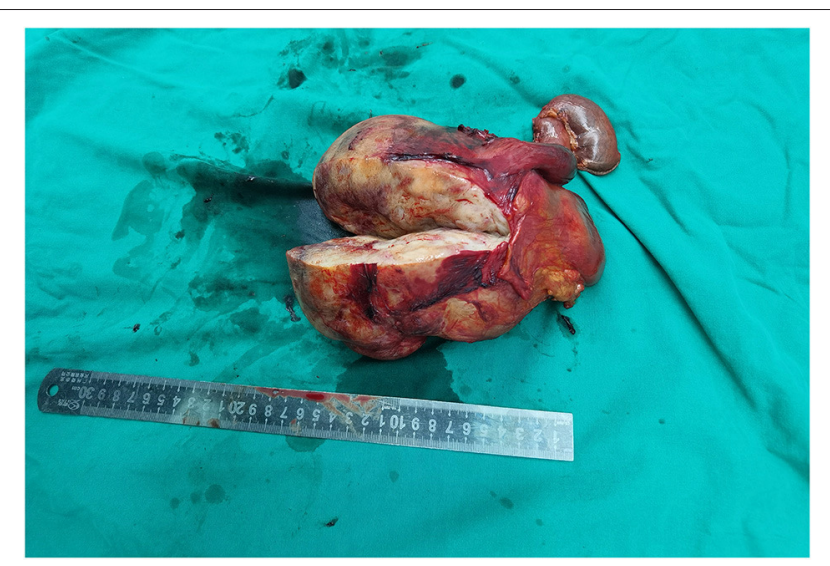

FIGURE 3 | The surgical specimen showed that the tumor was a well-delineated solitary mass, and that the actual size was $\sim 17 \times 12 \mathrm{~cm}$. The section of the tumor showed a pale and solid mass, which is relatively tough.

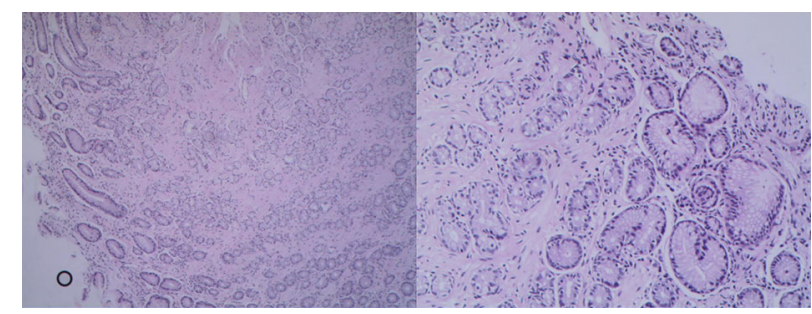

FIGURE 4 | Pathological images showed spindle cells with involvement of the pancreas, duodenal serosa, and serosa of the gallbladder. No lymph node metastasis was found

features. Complete surgery is the main treatment for intraabdominal spindle cell tumors. If the tumor invades other organs in the abdominal cavity, extensive radical resection may be carried out (15). In our case, the patient presented with a tumor with a maximum cross section diameter of $11 \mathrm{~cm}$, but we successfully removed the tumor and reconstructed the digestive tract. Recurrence rates as high as $20-68 \%$ have been reported even after negative-margin excision of desmoid tumors, and the prognostic significance of microscopic positive margin remains unknown (16). Through following the treatment model of surgically removing the entire tumor with an adequate tumorfree margin, we thoroughly detected the tumor and part of

\section{REFERENCES}

1. Suurmeijer AJH, Dickson BC, Swanson D, Zhang L, Sung YS, Cotzia P, et al. A novel group of spindle cell tumors defined by $\mathrm{S} 100$ and CD34 co-expression shows recurrent fusions involving RAF1, BRAF, and NTRK1/2 genes. Genes Chromosomes Cancer. (2018) 57:611-21. doi: 10.1002/gcc.22671

2. Michal M, Ptáková N, Martínek P, Gatalica Z, Kazakov DV, Michalová K, et al. S100 and CD34 positive spindle cell tumor with prominent perivascular hyalinization and a novel NCOA4-RET fusion. Genes Chromosomes Cancer. (2019) 58:680-5. doi: 10.1002/gcc.22758 the invading lesions, and to date the patient has been free of recurrence since the surgery.

In conclusion, due to being a rare disease, much more studies on retroperitoneal spindle cell tumors are needed to enhance the management of this lesion. Complete resection is the gold standard for this kind of rare disease.

\section{DATA AVAILABILITY STATEMENT}

The raw data supporting the conclusions of this article will be made available by the authors, without undue reservation.

\section{ETHICS STATEMENT}

Written informed consent was obtained from the individual(s) for the publication of any potentially identifiable images or data included in this article.

\section{AUTHOR CONTRIBUTIONS}

$\mathrm{HH}$ prepared and wrote this article. ZD, HX, and LL were involved in managing the patient. ZC prepared the intraoperative pictures. CY revised the manuscript as well as acted as the corresponding author. $\mathrm{ZH}$ wrote and reviewed this manuscript. SZ and CY were the main surgeon. CS was involved directly in managing the patient. All authors contributed to the article and approved the submitted version.

\section{FUNDING}

This research was financially supported by the funds from Science and Technology Planning Project of Guizhou Province, Science and Technology Cooperation Support of Guizhou Province [2021] General 080, Clinical Medical Research Center of Hepatobiliary Surgery of Guizhou Province, Science and Platform Talent of Guizhou Province [2017], Science and Technology Foundation of Health and Family Planning Commission of Guizhou Province [grant number: gzwkj2021167], and Science and Technology Planning Project of Guizhou Province construction contract [2019].

\section{ACKNOWLEDGMENTS}

We would like to thank CY for his guidance on this article and for editing and proofreading this English manuscript.

3. Fisher C. Immunohistochemistry in diagnosis of soft tissue tumours. Histopathology. (2011) 58:1001-12. doi: 10.1111/j.1365-2559.2010. 03707.x

4. Ellis GL, Langloss JM, Heffner DK, Hyams VJ. Spindle-cell carcinoma of the aerodigestive tract. An immunohistochemical analysis of 21 cases. Am J Surg Pathol. (1987) 11:335-42. doi: 10.1097/00000478-19870500000001

5. Lade H, Gupta N, Singh PP, Dev G. Spindle-cell hemangioendothelioma of the posterior pharyngeal wall. Ear Nose Throat J. (2005) 84:3625. doi: $10.1177 / 014556130508400616$ 
6. Abdullah JM, Mutum SS, Nasuha NA, Biswal BM, Ariff AR. Intramedullary spindle cell hemangioendothelioma of the thoracic spinal cord-case report. Neurol Med Chir (Tokyo). (2002) 42:259-63. doi: 10.2176/nmc.42.259

7. Rakheja D, Molberg KH, Roberts CA, Jaiswal VR. Immunohistochemical expression of beta-catenin in solitary fibrous tumors. Arch Pathol Lab Med. (2005) 129:776-9. doi: 10.5858/2005-129-776-IEOCIS

8. Bhattacharya B, Dilworth HP, Iacobuzio-Donahue C, Ricci F, Weber K, Furlong MA, et al. Nuclear beta-catenin expression distinguishes deep fibromatosis from other benign and malignant fibroblastic and myofibroblastic lesions. Am J Surg Pathol. (2005) 29:653-9. doi: 10.1097/01.pas.0000157938.95785.da

9. Saito T, Oda Y, Tanaka K, Matsuda S, Tamiya S, Iwamoto Y, et al. Beta-catenin nuclear expression correlates with cyclin D1 overexpression in sporadic desmoid tumours. J Pathol. (2001) 195:222-8. doi: 10.1002/path.942

10. Yamaguchi U, Hasegawa T, Masuda T, Sekine S, Kawai A, Chuman H, et al. Differential diagnosis of gastrointestinal stromal tumor and other spindle cell tumors in the gastrointestinal tract based on immunohistochemical analysis. Virchows Arch. (2004) 445:142-50. doi: 10.1007/s00428-0041055-8

11. Torres JC, Xin C. An unusual finding in a desmoid-type fibromatosis of the pancreas: a case report and review of the literature. J Med Case Rep. (2018) 12:123. doi: 10.1186/s13256-018-1635-x

12. Carlson JW, Fletcher CD. Immunohistochemistry for beta-catenin in the differential diagnosis of spindle cell lesions: analysis of a series and review of the literature. Histopathology. (2007) 51:509-14. doi: 10.1111/j.1365-2559.2007.02794.x

13. Lacka DE, Nasierowska-Guttmejer A. Fibromatosis - immunohistochemical evaluation, differential diagnosis from gastrointestinal tumors, and other mesenchymal tumours. Prz Gastroenterol. (2019) 14:79-85. doi: 10.5114/pg.2019.83429
14. Deyhimi P, Arefian MR, Mahzouni P. Comparative analysis of the histopathologic features, $\beta$-catenin, and Ki67 expression between fibromatosis and fibrosarcoma. J Dent (Shiraz). (2019) 20:255-63. doi: 10.30476/dentjods.2019.44900

15. Lev D, Kotilingam D, Wei C, Ballo MT, Zagars GK, Pisters PW, et al. Optimizing treatment of desmoid tumors. J Clin Oncol. (2007) 25:178591. doi: 10.1200/JCO.2006.10.5015

16. Ballo MT, Zagars GK, Pollack A, Pisters PW, Pollock RA. Desmoid tumor: prognostic factors and outcome after surgery, radiation therapy, or combined surgery and radiation therapy. J Clin Oncol. (1999) 17:15867. doi: 10.1200/JCO.1999.17.1.158

Conflict of Interest: The authors declare that the research was conducted in the absence of any commercial or financial relationships that could be construed as a potential conflict of interest.

Publisher's Note: All claims expressed in this article are solely those of the authors and do not necessarily represent those of their affiliated organizations, or those of the publisher, the editors and the reviewers. Any product that may be evaluated in this article, or claim that may be made by its manufacturer, is not guaranteed or endorsed by the publisher.

Copyright (c) 2021 Hua, He, Lei, Xie, Deng, Cheng, Zuo, Sun and Yu. This is an open-access article distributed under the terms of the Creative Commons Attribution License (CC BY). The use, distribution or reproduction in other forums is permitted, provided the original author(s) and the copyright owner(s) are credited and that the original publication in this journal is cited, in accordance with accepted academic practice. No use, distribution or reproduction is permitted which does not comply with these terms. 